MAGNETOHYDRODYNAMICS Vol. 54 (2018), No. 1-2, pp. 127-129

DOI: $10.22364 / \mathrm{mhd} .54 .1-2.22$

\title{
APPLICATION OF MAGNETIC NANOSCALE DISPERSED SYSTEMS IN ELECTROMECHANICAL DEVICES OPERATED AT SEPARATIONS OF GAS AND LIQUID FIRE EXTINGUISHING AGENTS
}

\author{
A.P. Sizov, D.S. Repin, V.S. Elovsky, V.A. Komelkov, A.V. Toporov \\ Ivanovo Fire and Rescue Academy of the State Fire Service of EMERCOM of Russia, \\ 33 Stroiteley pr., Ivanovo, Russia
}

Stringent demands to the durability and operating conditions have been imposed on the electromechanical devices with magnetic fluids when introducing these into practice. Therefore, the problem of implementation was solved by combining the production of MFs and the design of sealing devices. An experimental sample made in accordance with the design presented in [1] was investigated.

Electromechanical devices are used in various gas environments. Their operating parameters and durability depend on the processing medium. This often creates a neutral medium based on the maximum durability of the unit. This type of medium may be the air or neutral gases. In this case, the operational and environmental situation can be of different nature, temperature and pressure. Therefore, it is necessary to create conditions to prevent the mutual penetration of gases. The elements of the electromechanical devices must have additional sealing to prevent penetration of one medium into another. The problem for such seals turns out to be complicated if the element to be sealed is mobile. The sealing of movable elements is achieved in various ways. The most widely used sealers are the sealers for rotating shafts of various machines. Such devices are operated under different conditions: in a wide range of temperatures, under the influence of vibration, acceleration, radiation in all cases, they have to be efficient.

One of the complex problems of sealing the shafts was the separation of vacuum and air. In this case, the sealing device must not allow the gas to penetrate the vacuum and, at the same, time it must have its own low losses due to friction. With reference to the analysis of the sealing technology, it has been concluded that it is necessary to use a drop medium as a sealer and to keep it in the gap between the shaft, and predetermined element magnetic forces must be used. Such a seal was the magnetic fluid (MF).

Stringent demands to the durability and operating conditions have been imposed on the electromechanical devices with magnetic fluids when introducing these into practice. Therefore, the problem of implementation was solved by combining the production of MFs and the design of sealing devices. An experimental sample made in accordance with the design presented in [1] was investigated.

The investigations of the parameters of the developed and produced magnetic fluid seal (MFS) were performed in a specially designed setup (Fig. 1) consisting of a vacuum unit VU-05-4 PR 1, a pre-vacuum pump 2 and a tested MFS 3. An annular chamber for coolant circulation was installed in the MFS housing. The chamber was connected to a thermostat or to a Dewar vessel. The MFS leakage reliability was investigated using the developed setup.

The MFS was filled with a magnetic fluid and installed at the flange of the vacuum chamber in which a vacuum was given rise. The velocity in this sealing 


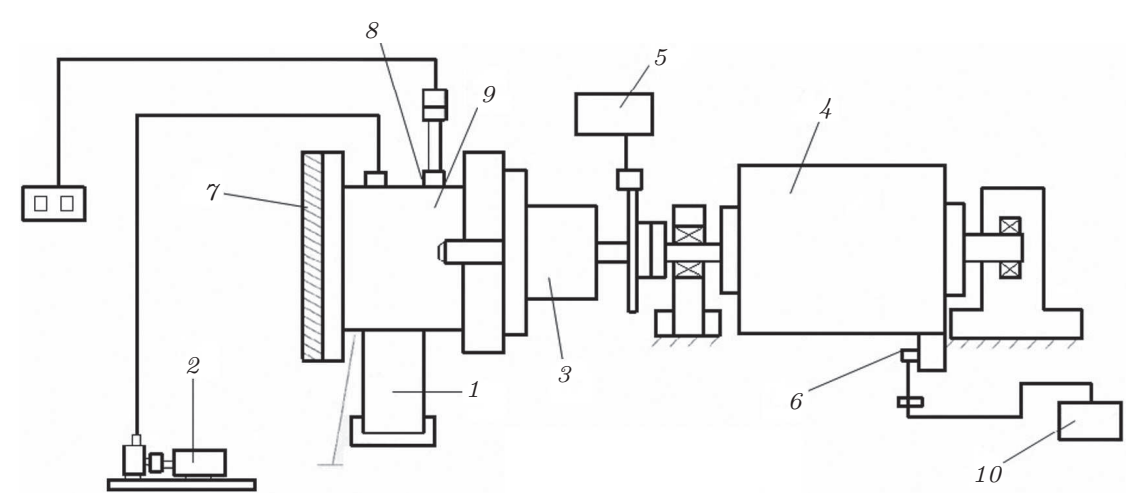

Fig. 1. Experimental setup. 1 - vacuum unit VU-05-4 PR; 2-pre-vacuum pump; 3 tested MFS; 4-drive motor; 5 - rotational velocity sensor of the MFS shaft; 6 - strain gauge transducer; 7 -plug of the vacuum unit; 8 - pressure sensor; 9 -vacuum chamber; 10 - strain gauge; 11 - vacuum gauge.

was varied by a drive motor, and the required temperature of the MFS shell was supported by pumping the coolant through the annular chamber. The positive temperatures were maintained by means of pumping a liquid coolant through the chamber coming from the thermostat; the negative temperatures in the housing were created by pumping liquid nitrogen through the chamber. The tightness of the MFS under specified conditions was determined by the change in magnitude of the vacuum in the chamber 9 when the chamber was disconnected from the vacuum source in this chamber by shutting the chambers damper.

With reference to the obtained experimental results, it was concluded that the MFS has a high leakproofness [2]. The performed numerous theoretical and practical studies of the rheological parameters of the MFs subject to magnetic fields in a specially designed apparatus have revealed a significant impact of $C_{\mathrm{v}}$ ferroparticles on the physical properties of the MF concentration. It was found that when $C_{\mathrm{v}}$ was greater than some optimal value $C_{\mathrm{v}}$ opt., the MF properties were similar to those of Weissenberg liquids [3]. Such studies made it possible to synthesize a magnetic fluid which ensures the maximum life period for the MFS.

The results show that the magnetic fluids can be used as a sealing material for electromechanical devices in the gas fire extinguishing installations. In the rescue equipment, water is widely used as a fire-extinguishing agent. It is supplied through fire pumps. To enhance their durability, the MFS must be capable of operating in contact with water. The study of the magnetic-seal characteristics has shown that the sealing process is different when sealing a liquid medium from a gaseous medium [4]. The MFS in the liquid medium has a threshold velocity above which the sealing capacity is impaired, and when the rotation rate decreases below the threshold, the sealing capacity is restored.

The leakage through the MFS was observed as a series of drops. Therefore, to seal water, it was suggested to use a combined seal in which the contact of the liquid crystal with the sealing medium was restricted. As such limiting element, it was proposed to use an end seal which was introduced into the design of the MFS. The manufacturing of the mechanical seal required [5] a high-cost preparation of friction pairs. Therefore, it is suggested to use the MF for mechanical seals as a lubricant and locking liquid.

Such seals can solve the problems of pumps in standby and dynamic modes. 
Application of magnetic nanoscale dispersed systems in electromechanical ...

\section{References}

[1] R.E. Rosenzweig. Ferrohydrodynamics (Translation from English, ed. V.V. Gogosov, Moscow, Mir Publishing House, 1989), 356 p.

[2] L.A. Kondakov,A.I. Golubev. Seals and others. Sealing and Sealing Equipment (Reference book, Eds. A.I. Golube, L.A. Kondakov, Mechanical Engineering, 1986), pp. 32-33.

[3] B.N. Berkovsky, A.N. Vislovich, A.S. Larin. Some problems of the static and dynamic interaction of ferrofluids with solid bodies. In: Problems of Mechanics of Magnetic Fluids (Minsk, BSSR ITMO AS, 1981), pp. 14-36.

[4] A.R. Loginov, B.Y. SacheK. The installation for material testing for friction and wear in ferromagnetic fluid medium. In: Proc. the IV All-Union Conference on Magnetic Fluids. Ferromagnetic Masses (Ivanovo Power Engineering Institute, 1985), vol. 1 (in Russian).

[5] A.R. Loginov, B.Y.SacheK, I.V. Karagelsky. Study of burnishing bronze-steel pair in ferromagnetic fluid medium.. In: Proc. the IV All-Union Conference on Magnetic Fluids. Ferromagnetic Masses (Ivanovo Power Engineering Institute, 1985), vol. 1 (in Russian).

Received 27.12.2017 